\title{
Lectura e interpretación de gráficos estadísticos, ¿cómo lo hace el ciudadano?
}

\author{
Audy Salcedo \\ audy.salcedo@ucv.ve \\ https://orcid.org/0000-0002-9783-8509 \\ Universidad Central de Venezuela (UCV); Caracas, Venezuela \\ Universidad Católica del Maule (UCM) \\ Talca, Chile \\ Jesús González \\ jagonza05@gmail.com \\ https://orcid.org/0000-0002-7249-9264 \\ Universidad Central de Venezuela (UCV) \\ Caracas, Venezuela \\ Johnnalid González \\ estadisticaucv.2012@,gmail.com \\ https://orcid.org/0000-0001-6537-3893 \\ Universidad Central de Venezuela (UCV) \\ Caracas, Venezuela
}

Recibido: 05/09/2020 Aceptado: 11/02/2021

\section{Resumen}

La lectura, interpretación y evaluación de gráficos estadísticos forma parte de las competencias que debe alcanzar un ciudadano común para actuar con prestancia frente a la información estadística que a diario se encuentra en distintos medios. Estas razones han llevado a la mayoría de los países a incluir temas de estadística desde la educación primaria. El objetivo de esta investigación es analizar cómo leen e interpretan gráficos estadísticos una muestra de ciudadanos venezolanos. A partir de un gráfico estadístico, se elaboraron cuatro preguntas de diferentes niveles de exigencia. El cuestionario fue respondido por 407 venezolanos con diferentes niveles de formación académica. Los resultados evidencian que el grupo estudiado no tiene dificultades para leer de forma literal el gráfico, pero tiene problemas cuando se le pide reportar una información no explícita o generar una nueva información. Estos resultados pueden ser una referencia para la revisión de los procesos de formación estadística del ciudadano.

Palabras clave: Comprensión de gráficos estadísticos; Ciudadanía; Representación de datos; Alfabetización estadística.

\section{Leitura e interpretação de gráficos estatísticos, como o cidadão o faz?}

\section{Resumo}

A leitura, interpretação e avaliação de gráficos estatísticos fazem parte das competências que um cidadão comum deve adquirir para atuar com excelência perante a informação estatística que se encontra diariamente nos diversos meios de comunicação. Por razões como essas, a maioria dos países incluiu tópicos estatísticos desde a educação primária. O objetivo desta pesquisa é analisar como uma amostra de cidadãos venezuelanos lê e interpreta gráficos 
estatísticos. A partir de um gráfico estatístico, serão elaboradas quatro questões de diferentes níveis de demanda. O questionário foi respondido por 407 venezuelanos com diferentes níveis de formação acadêmica. Os resultados mostram que o grupo estudado não tem dificuldade em ler o gráfico literalmente, mas tem problemas quando solicitado a relatar informações não explícitas ou gerar novas informações. Esses resultados podem servir de referência para a revisão dos processos de formação estatística do cidadão.

Palavras chave: Compreensão de gráficos estatísticos; Cidadania; Representação de dados; Letramento estatístico.

\title{
Reading and interpretation of statistical graphs, how does the citizen do it?
}

\begin{abstract}
Reading, interpreting and evaluating statistical graphs is part of the competences that an ordinary citizen must achieve to confront with excellence the statistical information that is daily found in different media. For this reason, most countries have included statistical topics since primary education. The objective of this research is to analyze how a sample of Venezuelan citizens read and interpret statistical graphics. From a statistical graph, four questions of different levels of demand were elaborated. The questionnaire was answered by 407 Venezuelans with different levels of academic training. The results show that the studied group does not have difficulties to literally read the graph, but has problems when asked to report non-explicit information or to generate new information. These results can be a reference for the review of the citizen's statistical training processes.
\end{abstract}

Keywords: Comprehension of statistical graphics; Citizenship; Data representation; Statistical literacy.

\section{Introducción}

Una característica de la sociedad de la información y del conocimiento son las grandes masas de datos, que son analizadas y transformadas en información para la toma de decisiones, o para producir conocimiento. Esta situación ha generado que los profesionales de la estadística tengan alta demanda para formar equipos interdisciplinarios y trabajar en el análisis de datos.

Desde hace ya varias décadas, la mayoría de los países han incluido a la estadística como uno de los componentes de la formación general de todo ciudadano (Ben-Zvi \& Makar, 2016). Para Batanero \& Borovcnik (2016) la estadística y la probabilidad deben incluirse en la formación de todo ciudadano por tres razones: (a) son esenciales para el razonamiento crítico, (b) tienen un rol instrumental en otras disciplinas, (c) son fundamentales para la planificación y la toma de decisiones en muchas profesiones. Con la inclusión de temas de estadística y probabilidad desde los primeros grados no se pretende convertir a todos los ciudadanos en estadísticos; lo que se desea es que alcancen la alfabetización estadística, uno de cuyos 
elementos es la compresión e interpretación de gráficos estadísticos (Gal, 2002; Garfield \& BenZvi, 2007). Dichos gráficos aparecen en diarios, revistas, redes sociales, noticieros de televisión y otros medios de comunicación, para ofrecer información a los ciudadanos. Los gráficos pueden ayudar a observar aspectos de la distribución (por ejemplo, valores atípicos) que podrían pasar inadvertidos con el cálculo de medidas de estadística descriptivas (Leavy, 2006; Tukey, 1977).

Pero comprender e interpretar un gráfico no es una actividad trivial. La investigación indica que son frecuentes las dificultades y errores en la interpretación de gráficos Fernández, García-García, Arredondo, \& López, 2019 o Monteiro \& Ainley, 2007). A las dificultades propias de la compresión de gráficos, hay que agregar el caso de gráficos estadísticos que, con intención o no, son manipulados para expresar una idea que no reflejan los datos (GarzónGuerreo, 2020; Sutherland \& Ridgway, 2017). Si bien organismos como el Common Core State Standards Initiative (2010) recomiendan la inclusión del estudio de gráficos estadísticos desde la educación primaria, con miras a ayudar al ciudadano a desarrollar una postura crítica respecto a la estadística, Engel (2019) considera que es importante enfocarse en temas de relevancia social y propone una subdisciplina que denomina Estadística Cívica. La Estadística Cívica busca obtener significados a partir de datos reportados sobre los procesos sociales, el bienestar social y económico, así como los derechos civiles. Comprender esos temas es de crucial importancia para la participación cívica en las sociedades modernas, en temas de interés general como es el caso del COVID-19, que ha generado una gran cantidad de información estadística.

En este trabajo se analizan cómo comprenden e interpretan gráficos estadísticos un grupo de ciudadanos venezolanos egresados de la educación media, con formación parcial o total en educación superior. En particular nos interesamos por los niveles de lectura que muestran estos ciudadanos al interpretar un gráfico de barras. Aunque en la revisión bibliográfica realizada se encontraron diferentes trabajos donde se analizan los niveles de lectura vinculados a un gráfico, no se encontraron investigaciones en Venezuela donde se pida a ciudadanos que analicen gráficos estadísticos. Por ello, se considera que la información de este estudio puede ser una referencia para la revisión de los procesos de formación estadística del ciudadano venezolano.

\section{Los gráficos estadísticos en la formación del ciudadano en Venezuela}


Los inicios de la formación estadística en Venezuela están vinculados con el censo de población y vivienda. En 1937 se dictó, en la Universidad Central de Venezuela (UCV), el Curso de Capacitación Estadística con el objetivo de formar personal que cumpliera las labores que se desprendían de la Ley de Estadística y Censos Nacionales. En 1939 se iniciaron los estudios estadísticos en la Escuela de Preparación Estadística, cuya función era la preparación de personal a nivel medio para la estadística y el censo. En 1953 se crea la Escuela de Estadística y Ciencias Actuariales de la UCV (Guardia de Sanz, 1988). Por tanto, como en la mayoría de los países, la estadística se comenzó a estudiar en Venezuela en la educación superior.

Es a partir de 1972 cuando se introducen, en los programas de matemática de quinto año de educación media, nociones de probabilidad y estadística. Esos contenidos se mantuvieron iguales en el ajuste realizado en 1990 (Ministerio de Educación, 1990). Con la promulgación de la Ley Orgánica de Educación de 1980, se crea la Educación Básica (E.B.) de nueve grados y se hace un cambio curricular en matemática. En ese cambio, la estadística y la probabilidad se incluyen desde los niveles iniciales de la educación venezolana, la estadística se comienza a estudiar desde primer grado y la probabilidad desde quinto (Ministerio de Educación, 1987).

A finales de los 90, se produce un nuevo cambio curricular, centrado en el desarrollo de competencias y se modificaron los programas de matemáticas de los seis primeros grados. Los contenidos de estadística se mantienen desde primer grado, pero los de probabilidad ahora se inician en segundo (Ministerio de Educación, 1997). Se tenía previsto que ese cambio curricular provocara modificaciones en los planes de estudio de los otros grados, pero no se continuó. En consecuencia, los programas de matemáticas en Venezuela son de tres momentos distintos: primaria ( $1^{\circ}$ a $6^{\circ}$ grado) al currículo de 1997 , mientras los tres primeros años de bachillerato $\left(7^{\circ}\right.$, $8^{\circ}$ y $9^{\circ}$ de la antigua E.B.) a los programas de 1987 y dos últimos años del bachillerato de 1990.

La anterior aclaración es importante para entender la falta de continuidad en el estudio de gráficos estadísticos entre la primaria y secundaria venezolana. En primaria los gráficos estadísticos aparecen en todos los grados, con cambios en el nivel de las destrezas que se aspira a desarrollar. Se trabajan con pictogramas ( $1^{\circ}$ a $4^{\circ}$ grado), gráficos de barras $\left(2^{\circ}\right.$ a $6^{\circ}$.), histogramas y gráficos de línea ( $5^{\circ}$ a 6 to.), sectores $\left(6^{\circ}\right)$. Desde los primeros grados se trabaja con la recolección, organización y representación de datos tanto en tablas como en gráficos, además de interpretar los datos graficados. Los datos a graficar deben ser recolectados por los estudiantes y provenientes de distintas situaciones (sociales, ambientales, sanitarias, deportivas, 
entre otras) y de contextos significativos para ellos. Se aspira que en $6^{\circ}$ grado el estudiante pueda a elegir el gráfico más adecuado para organizar la información, según la naturaleza de los datos. En general, en ese nivel, el estudio de gráficos se corresponde con recomendaciones de investigadores (Franklin et al., 2005; Friel et al., 2001).

En la educación secundaria, los gráficos solo aparecen en el primer año, donde se trabaja el histograma, centrando la atención en la construcción e interpretación. En los años subsiguientes se hace énfasis en el cálculo de medidas de tendencia central y de dispersión. Lo que se pudo avanzar sobre los gráficos estadísticos durante la primaria queda interrumpido, por tratarse de programas diseñados en distintos momentos. La discontinuidad en el estudio de los gráficos pudo subsanarse, en parte, con los libros de matemáticas de la Colección Bicentenario, diseñados por el Ministerio del Poder Popular para la Educación, pero, al contrario, la profundizó, pues ellos solo responden parcialmente a los programas vigentes. Respecto a los gráficos estadísticos, los libros de primaria trabajan casi exclusivamente la construcción de gráficos de barra, dejando de lado la interpretación. El pictograma y el histograma solo se estudian en un grado, mientras que los gráficos de línea y de sectores fueron excluidos (Salcedo, 2017). Adicionalmente, muchos docentes señalan que el tiempo no les permite trabajar en el aula los temas de estadística y probabilidad, los cuales están ubicados al final de los planes de estudio.

A nivel universitario, la estadística descriptiva, incluyendo los gráficos estadísticos, suelen ser el punto común en la mayoría de las carreras de pregrado en Venezuela, aunque en una buena parte de ellas también se estudian fundamentos de inferencia estadística. En postgrado, es frecuente encontrar seminarios de estadística, donde también se estudian los gráficos. Pareciera entonces que solo aquellos ciudadanos venezolanos que sigan estudios universitarios tienen una posibilidad real de profundizar en el estudio de los gráficos estadísticos y desarrollar la capacidad para interpretarlos y evaluarlos críticamente.

\section{Marco de referencia}

Aunque en la literatura abundan las definiciones de alfabetización estadística y no hay un consenso sobre su definición, en la mayoría de ellas incluyen la representación gráfica como un elemento básico de la misma. Además de los ya mencionados, también Engel (2019), Franklin et al. (2007) y Weiland (2017) destacan en el desarrollo de la alfabetización estadística. 
Cuando se habla de enseñanza de la estadística, se incluyen, además, los gráficos estadísticos como un componente de las ideas fundamentales en estadística (Batanero \& Borovcnik, 2016).

Batanero, Arteaga y Ruíz (2010) señalan que un gráfico estadístico es un objeto semiótico complejo, pues al leerlo se debe hacer la traducción y comprensión del gráfico en sí, y también de cada pieza de información que lo acompaña. Curcio (1987) señala que la comprensión del gráfico depende de tres factores: (a) los conocimientos previos sobre el tema que se representa mediante la gráfica; (b) el conocimiento de los conceptos matemáticos involucrados, (c) el conocimiento de los convencionalismos para la construcción de los gráficos. Entonces, para comprender e interpretar un gráfico es necesario la lectura por separado de cada una de sus partes, hacer interrelaciones entre las variables representadas y luego vincularlo con el contexto para producir conclusiones sobre la situación estudiada.

Friel, Curcio \& Bright (2001) definen la comprensión gráfica como las habilidades de los lectores para derivar significados de gráficas creadas por otros o por ellos mismos. Los autores y sus colaboradores identifican cuatro niveles de comprensión de un gráfico (Curcio, 1989 y Friel et al., 2001):

- Leer los datos. Se hace la lectura literal de los valores del gráfico estadístico, no se realiza comparaciones ni se produce nueva información.

- Leer entre los datos. Se comparan e interpretan los valores del gráfico para producir una información no explícita en él. Se buscan relaciones entre las cantidades, y para ello se pueden comparar a simple vista o realizar cálculos sencillos. Exige comprender la estructura del gráfico y las relaciones contenidas en él.

- Leer más allá de los datos. A partir del gráfico se hacen predicciones o inferencias de valores a partir de la información mostrada en el gráfico. Requiere la extrapolación de datos, e inferir a partir de los valores del gráfico información que está presente de forma implícita en él. Es necesario conocer el contexto en que se presentan los datos.

- Leer detrás de los datos. Demanda mirar críticamente el uso del gráfico y conectar la información gráfica con el contexto. Se evalúan los datos en su contexto, pero además se examinan la calidad de los datos, y la metodología usada para su recolección. 
Aoyama (2007) propone un modelo jerárquico basado en la taxonomía SOLO para evaluar la comprensión de las gráficas e identifica cinco niveles de interpretación de gráficas para los estudiantes:

- Idiosincrático. El estudiante no puede leer valores o tendencias en el gráfico o proporcionar valores incorrectos cuando lo lee. No conecta las características extraídas del gráfico con el contexto.

- Lectura básica del gráfico. El estudiante puede leer valores y tendencias en el gráfico, pero no puede explicar los significados contextuales de las tendencias o características que observa en los datos. No relaciona el gráfico con el contexto.

- Relacional. El estudiante puede leer valores particulares y tendencias, así como explicar los significados contextuales en función de las características mostradas en el gráfico. No obstante, no puede sugerir interpretaciones alternativas, usa exclusivamente los significados presentados.

- Crítico. El estudiante puede leer el gráfico y comprender las variables contextuales presentadas, además, puede evaluar la confiabilidad del significado contextual descrito en el gráfico. Puede cuestionar la información presentada.

- Hipotetizador: El estudiante puede leer el gráfico y evaluar la información presentada, además puede formular sus propias hipótesis explicativas o modelos. No es solo un receptor de información; actúa como investigador estadístico.

Los trabajos de Aoyama (2007) y Friel et al. (2001) pueden ser punto de apoyo para planificar la enseñanza de gráficos estadísticos en los distintos niveles de la educación.

\section{Algunos antecedentes}

Las investigaciones reportan que comprender y extraer información de un gráfico no suele ser una tarea sencilla. Por ejemplo, Arredondo, García-García y López (2019), Fernández, García-García, Arredondo, y López (2019), Gea, Arteaga y Cañadas (2017), Inzunsa (2015) y Wu (2004) describen los problemas en la interpretación y extracción de información del gráfico. En la mayoría de estos trabajos, los estudiantes se ubican en los niveles bajos de compresión de gráficos de la taxonomía utilizada. 
Wu (2004) señala que a los estudiantes de su muestra (de 13 a 15 años) les fue mejor en las tareas de lectura que en las tareas de interpretación y evaluación de gráficos. Monteiro y Ainley $(2006,2007)$ encuentran que futuros maestros de Brasil e Inglaterra analizan los gráficos sobre la base de su experiencia y opiniones personales, con poca referencia a los datos del gráfico. Parece que le dan preponderancia al conocimiento que tienen del contexto social de donde provienen los datos y dejan de lado las relaciones que se muestran en el gráfico.

Aoyama y Stephens (2003) trabajaron con estudiantes de dos escuelas públicas de Japón, 17 de quinto grado y 38 de octavo. Les pidieron analizar dos gráficos, de línea y de barras, para evaluar la capacidad de los estudiantes para crear nueva información. Los estudiantes debían estudiar por separado cada gráfico y luego analizarlos en conjunto a partir de una afirmación sobre la información presentada. Las respuestas al análisis de los gráficos por separado fueron analizadas según los niveles de compresión de gráficos propuesta por Friel et al. (2001) y la mayoría de los estudiantes de ambos grados se ubicó en el nivel Leer más allá de los datos. La taxonomía SOLO se usó para analizar las respuestas al análisis conjunto de los gráficos. La mayoría de los estudiantes de quinto grado se agruparon en los dos niveles más bajos de esa taxonomía, mientras que los de octavo grado se ubican en el segundo y tercer nivel.

Eudave (2009) realizó un estudio con jóvenes y adultos que cursaban la primaria o secundaria en alguna de las modalidades de educación para adultos. Entrevistó a 28 personas de distintas edades, medios y centros de estudio de México, y les planteó la tarea de analizar e interpretar una tabla y una gráfica de líneas, contestando preguntas que permitían evaluar los tres niveles iniciales de la taxonomía de Curcio (1987). Encontró que solo cinco personas hicieron la lectura adecuada de la tabla y el gráfico, por lo que se considera que alcanzaron los tres niveles de comprensión evaluados. Inzunsa (2015) presentó a estudiantes, de pregrado y maestría, cuatro gráficas con información demográfica y socioeconómica de boletines oficiales mexicanos y les pidió que las interpretaran. La mayoría de los estudiantes se ubicó primordialmente en los niveles idiosincrático y lectura básica, los más bajos del modelo de Aoyama (2007).

Gea et al. (2017) evaluaron la interpretación de gráficos estadísticos por parte de futuros profesores de matemáticas, quienes debían interpretar un histograma, un diagrama acumulativo y un gráfico de la caja. La mayoría de los estudiantes proporcionaron respuestas correctas, pero se ubicaban en niveles iniciales de la taxonomía de Friel et al. (2001): leer los datos y leer entre 
los datos. Solo en la interpretación del diagrama de caja hubo una mayor proporción de estudiantes en el nivel lectura más allá de los datos.

Arredondo et al. (2019), Fernández et al. (2019) y García-García, López Calvario y Arredondo (2018), trabajaron con estudiantes universitarios para analizar las interpretaciones de una tabla y un gráfico. En el primer trabajo se usó un gráfico de barras, en el segundo un gráfico de líneas, mientras que el primero se utilizó un gráfico circular. Las tres investigaciones reportan que la mayor proporción de los participantes alcanzó un nivel intermedio de lectura del gráfico, según la taxonomía utilizada en cada caso. En Arredondo et al. (2019) también se apoyaron en los niveles de comprensión gráfica de Friel et al. (2001), mientras que Fernández et al. (2019) trabajaron con una propuesta jerárquica diseñada a partir de las taxonomías de Friel et al. (2001) y Aoyama (2007). García-García et al. (2018) analizaron la lectura de los estudiantes apoyados en los niveles de comprensión de Curcio (1989) y Friel et al. (2001) y la jerarquía de Aoyama (2007) para la valoración crítica de la información

Garzón-Guerrero (2020) trabajó con 52 futuros docentes de educación primaria y secundaria de Italia (23) y Portugal (29) para estudiar su comprensión gráfica. A cada grupo le pidió analizar un gráfico de sectores, seleccionado de los medios de comunicación de su país. Los dos gráficos seleccionados tenían errores de construcción que podían provocar confusiones en su lectura e interpretación. Los resultados indican que los participantes presentaron dificultades en la interpretación y lectura completa y crítica del gráfico; sin diferencias estadísticamente significativas en las respuestas proporcionadas por las personas de ambos países. De acuerdo con las investigaciones antes citadas los grupos considerados tienen pocos problemas para hacer la lectura literal de los gráficos, así como hacer comparaciones, pero muestran dificultades para hacer inferencias a partir del gráfico o evaluarlo críticamente.

\section{Método}

El trabajo que presentamos es un estudio descriptivo, mediante encuesta, con diseño transeccional. La técnica de la encuesta permite hacer llegar el cuestionario a una mayor cantidad de ciudadanos venezolanos y el diseño transversal permite describir variables, y analizar su incidencia e interrelación en un momento dado, como en este caso, que se desea analizar cómo leen e interpretan gráficos estadísticos una muestra de ciudadanos. 


\section{Cuestionario}

Para recolectar la información se seleccionó una tarea tomada del informe PISA 2003 (INECSE, 2005) (Figura 2), que sería la tercera pregunta en y a partir de ella se elaboró un cuestionario de cuatro preguntas. La tarea seleccionada plantea una situación de educación, por lo que puede considerarse un contexto compresible para todos de los ciudadanos.

Figura 2. Pregunta 3 del Cuestionario

E1-gráfico-siguiente-muestra-los-resultados-en-un·examen-de-Ciencias para-dos

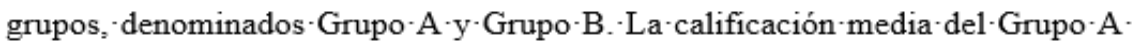

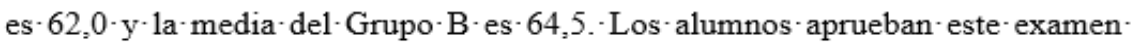
cuando-su-calificación·es·de·50·o más.ๆ

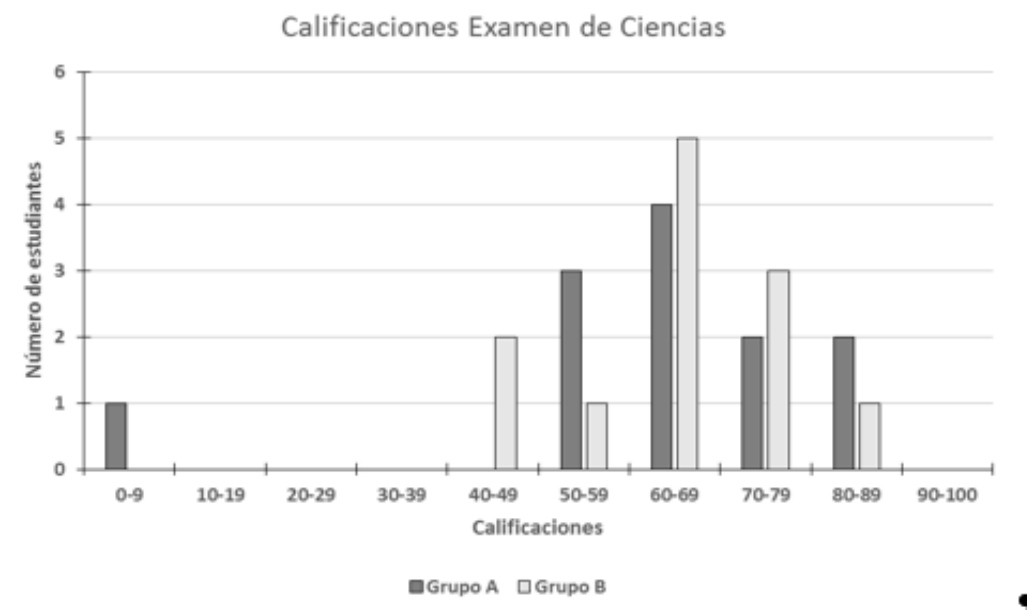

A1-observar-el-diagrama, -el-profesor-afirma-que, en-este-examen, -el-Grupo-B fue mejor-que-el-Grupo-A.ศ

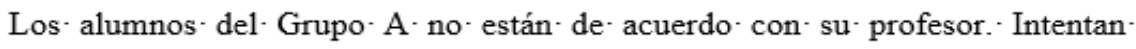
convencer- al' profesor- de- que- el- Grupo- B- no- tiene- por- qué haber- sido' necesariamente- el- mejor- en- este- examen.- Da- un- argumento- matemático, utilizando-la-información- del-diagrama, que- puedan- utilizar-los-alumnos-delGrupo-A. -

Fuente: INECSE (2005, p. 50)

Esta tarea fue clasificada en el estudio PISA de 2003 como de conexión, lo que significa que es un problema en un contexto familiar para el estudiante, aunque no es rutinario. Este tipo de problema exige establecer relaciones entre distintas representaciones de una misma situación o vincular diferentes aspectos de la información para hallar la solución (INECSE, 2005). En cuanto a la competencia matemática, es clasificada en el nivel 5. Se espera sea resuelta por 
estudiantes que pueden trabajar situaciones complejas, identificar condicionantes, reflexionar sobre sus acciones y comunicar sus interpretaciones y razonamientos. Las preguntas de PISA se clasifican en seis niveles de competencia matemática. El nivel más bajo es el 1, donde el estudiante debe llevar a cabo procedimientos rutinarios siguiendo instrucciones directas en situaciones explícitas. En el nivel 6, el estudiante debe formar conceptos, generalizar y utilizar información basada en investigaciones y modelos de situaciones de problemas complejos (OCDE, 2005). De acuerdo a los niveles de lectura de los gráficos de Friel et al. (2001), la tarea se clasifica como de Leer más allá de los datos. Para responderla con éxito las personas deben generar una nueva información a partir de los datos, porque esa información no se lee directamente del gráfico.

Antes de la tarea original de PISA (que constituyó la pregunta 3 del cuestionario) se agregaron dos preguntas de selección simple. Son preguntas de menor complejidad, donde se pide información que se puede leer directamente del gráfico o haciendo una sencilla operación matemática. El propósito es evaluar niveles más básicos de lectura del gráfico y dar oportunidad a los ciudadanos, que responden el cuestionario, de relacionarse de forma progresiva con la situación y el gráfico. Fueron las siguientes:

Pregunta 1. ¿Cuántos estudiantes del Grupo B obtuvieron entre 60-69 puntos?
a. 4
b. 5
c. 1
d. 3

Pregunta 2. ¿Cuántos estudiantes del Grupo A obtuvieron 59 puntos o menos?
a. 3
b. 1
c. 4
d. $\quad 7$

Usando la misma situación se agregó una cuarta pregunta (Figura 3), pero se formuló sobre un nuevo gráfico. La pregunta busca indagar si los estudiantes van más allá de la impresión inicial que ofrece el gráfico en cuanto a una amplia diferencia entre los dos grupos y contrastar la interpretación que hace el grupo A del gráfico con la información que proporciona. En una situación similar se podría encontrar un ciudadano cualquiera al leer un artículo en un medio de comunicación, donde debe verificar si la interpretación que se da en el artículo es congruente con el gráfico que apoya la información.

Al comienzo del cuestionario se incluyó el consentimiento informado. Se informaban los objetivos de la investigación, quienes la realizaban, la confidencialidad de la información proporcionada y cómo se usaría y el tiempo, aproximado, que llevaría responder las preguntas. 
Así mismo se informaba sobre su derecho a decidir participar o no en la investigación. Solo las personas que daban su consentimiento tenían acceso a las preguntas del cuestionario. Al final del cuestionario se incluyeron preguntas para recolectar información socio demográfica: sexo, edad y nivel de formación académica; las dos últimas se usaron en el análisis.

Figura 3. Pregunta 4 del cuestionario

Pregunta 4. Los estudiantes del Grupo A le pidieron al profesor que les proporcionara las calificaciones de todos los exámenes presentados por los dos grupos durante el año escolar. Con esa información calcularon el porcentaje de pruebas de cada grupo que habían aprobado todos los estudiantes y construyeron el siguiente gráfico.

\section{Porcentaje de Pruebas de Ciencias con Todos los Estudiantes Aprobados}

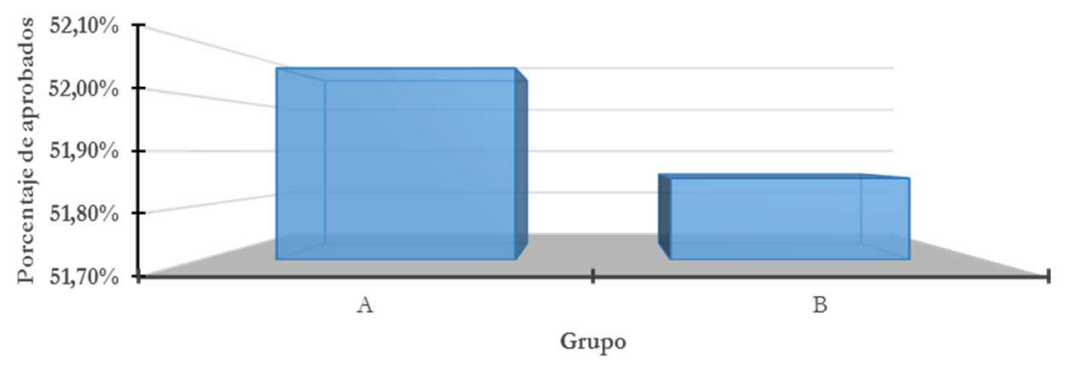

Los estudiantes del Grupo A mostraron al profesor el gráfico y afirman que su rendimiento es muy superior al del Grupo B. ¿Está de acuerdo con la afirmación del Grupo A? Argumenta tu respuesta.

$$
\text { Fuente: elaboración propia }
$$

\section{Procedimiento}

El cuestionario se publicó en la plataforma Google Forms y se utilizó un muestreo bola de nieve para hacer llegar el cuestionario a los potenciales participantes. Esa técnica de muestreo, no probabilística, es usada para acceder a poblaciones de baja incidencia y a individuos de difícil acceso por parte del investigador (Hernández Sampieri, Fernández, \& Baptista, 2006). En este caso se deseaba que respondieran ciudadanos en general, con diversidad de formación académica.

Los investigadores identificaron entre sus contactos un grupo pequeño de potenciales participantes, a los que enviaron el cuestionario por correo electrónico. A los que respondían el cuestionario se les pedía sugerir otras personas que podrían participar en la investigación. El procedimiento se repitió hasta conformar el grupo de cuestionarios que se analizan. El cuestionario estuvo disponible en Internet por 15 días. La decisión de cerrar la recepción de respuesta se tomó luego de percibir que en los últimos cinco días habían bajado de manera importante las respuestas. Se consideró que se habían agotado las posibilidades de ampliar la 
muestra. Los investigadores no tuvieron interacción con la muestra.

\section{Sujetos}

La muestra quedó conformada por 407 ciudadanos. La mayoría de sexo femenino (57,5\%), con un promedio de edad de 42,7 años y una desviación estándar de 15,9 años, lo cual sugiere que se trata de un grupo heterogéneo en cuanto a la edad (Min. 17, Máx. 85 años). En relación con su formación académica se tiene: Universitaria incompleta (23,6\%), Universitaria completa sin postgrado (23,6\%), con Especialización (17\%), con Maestría (25\%) y con Doctorado $(10,8 \%)$.

\section{Análisis de datos}

Las dos primeras preguntas eran de opción múltiple, en la que la persona elige una respuesta entre las prefijadas, por lo que solo recogió la frecuencia de cada alternativa. La tercera pregunta se evaluó según las indicaciones del estudio PISA, es decir, con el siguiente criterio:

Se da un argumento válido. Los argumentos válidos pueden estar relacionados con el número de estudiantes que aprueban, la influencia desproporcionada del caso extraño o el número de estudiantes con puntuaciones de nivel más alto. Se admiten como correctas las siguientes respuestas:

- Más alumnos en el Grupo A que en el Grupo B aprobaron el examen.

- Si ignoras al peor alumno del Grupo A, los alumnos del Grupo A lo han hecho mejor que los del Grupo B.

- Más alumnos del Grupo A que del Grupo B obtuvieron la puntuación de 80 o más. (INECSE, 2005, p. 50)

Las respuestas proporcionadas por los participantes fueron examinadas y clasificadas de forma individual e independiente por cada investigador. Se compararon las tres clasificaciones generadas y se discutieron para producir una única clasificación. Un procedimiento similar se utilizó con los argumentos proporcionados por los participantes en la pregunta 4, solo que en esta oportunidad las categorías surgieron a partir del análisis realizado durante la discusión de las tres clasificaciones individuales. Es importante acotar que algunas respuestas debieron leerse varias veces para poder clasificarlas. Incluso, en la discusión de las tres clasificaciones, hubo dudas con algunas respuestas sobre cuál categoría era más la pertinente para incluirla; en estos casos, siempre se optó por una categoría de consenso. 
Los datos fueron procesados con el programa estadístico Statistical Package for the Social Sciences. Se realizó un análisis exploratorio de datos, se calcularon medidas estadísticas descriptivas de las variables en estudio y se usó el estadístico Chi-cuadrado de Pearson para contrastar la hipótesis de homogeneidad de las respuestas según las edades y nivel de formación.

\section{Resultados}

\section{Lectura del gráfico (Preguntas 1 y 2)}

La primera pregunta estuvo dirigida a evaluar si los participantes pueden ubicar una de las categorías del gráfico y reportar la frecuencia. Se trata de la lectura literal del gráfico, sin interpretarlo, es el primer nivel, consistente solo Leer los datos según los niveles de lectura de Friel et al. (2001). El 93,6\% de los ciudadanos que participaron en el estudio señalaron la respuesta correcta, lo cual indica que fue una pregunta de baja dificultad para ellos. Entre las respuestas incorrectas la de mayor frecuencia fue la alternativa a), con 4,2\%, lo cual sugiere que identifican de forma adecuada el intervalo de la pregunta (60-69), pero leen la frecuencia del grupo A. Las otras dos alternativas reúnen porcentajes muy bajos: 1,5\% señala la alternativa c) y el $0,7 \%$ la d). Por tanto, los sujetos de la muestra no tuvieron dificultades en identificar al grupo B y su frecuencia en el intervalo solicitado. Esto coincide con lo señalado por Friel et al. (2001) en cuanto a que las preguntas donde los estudiantes hacer lectura literal del gráfico, en general, las resuelven sin inconvenientes.

Para responder de forma correcta la pregunta 2 se deben ubicar en el gráfico los intervalos donde el Grupo A obtuvo 59 puntos o menos, para luego sumar cada una de las frecuencias identificadas. No es una lectura directa como en la pregunta 1 , sino que se debe usar un procedimiento matemático para reportar la información solicitada. El 53,6\% de los participantes señala la respuesta correcta, lo cual sugiere ser una pregunta de mayor dificultad que la primera. El error más frecuente fue olvidar al estudiante del intervalo 0 a 9 y considerar como respuesta solo los estudiantes del grupo A en el intervalo 50-59. Por ello, señalan la opción d (3 estudiantes) como respuesta correcta, la cual reúne el 37,6\% del total de respuestas. Un $8,3 \%$ señala la alternativa b (un estudiante); esto podría significar que solo identifican al estudiante del intervalo 0 a 9 y no incluyen a los estudiantes del intervalo 50-59. El 0,5\% restante señala la alternativa d (siete estudiantes). Al igual que en la pregunta anterior, la mayoría de los participantes pueden ofrecer la respuesta correcta al identificar el grupo de interés en los 
intervalos requeridos. No obstante, un 46,4\% parece tener problemas con la condición de la pregunta, obtener 59 puntos o menos, ya que identifican solo una parte del grupo que cumple con esa premisa.

Este grupo de ciudadanos no tienen dificultades para leer de forma literal el gráfico (Leer los datos), pero evidencian problemas para reportar una información no explícita en el gráfico (Leer entre los datos). Un comportamiento similar se consigue en los grupos estudiados por Aoyama \& Stephens (2003), Arteaga et al. (2017), Gea et al. (2017) y Wu (2004), entre otros. Previamente lo informaban Friel et al. (2001), al afirmar: "los estudiantes experimentan pocas dificultades con las preguntas de leer los datos, pero cometen errores cuando se encuentran con preguntas de leer entre los datos" (p. 130). Esos errores parecen estar asociados a la lectura de los ejes o compresión de la escala (Cavalcanti \& Guimarães, 2019; Friel et al. 2001).

\section{Argumentos de los participantes (preguntas 3 y 4)}

En la Figura 4 se presentan los porcentajes de respuestas alcanzados en la pregunta 3.

Figura 4. Porcentaje de respuestas a la pregunta 3

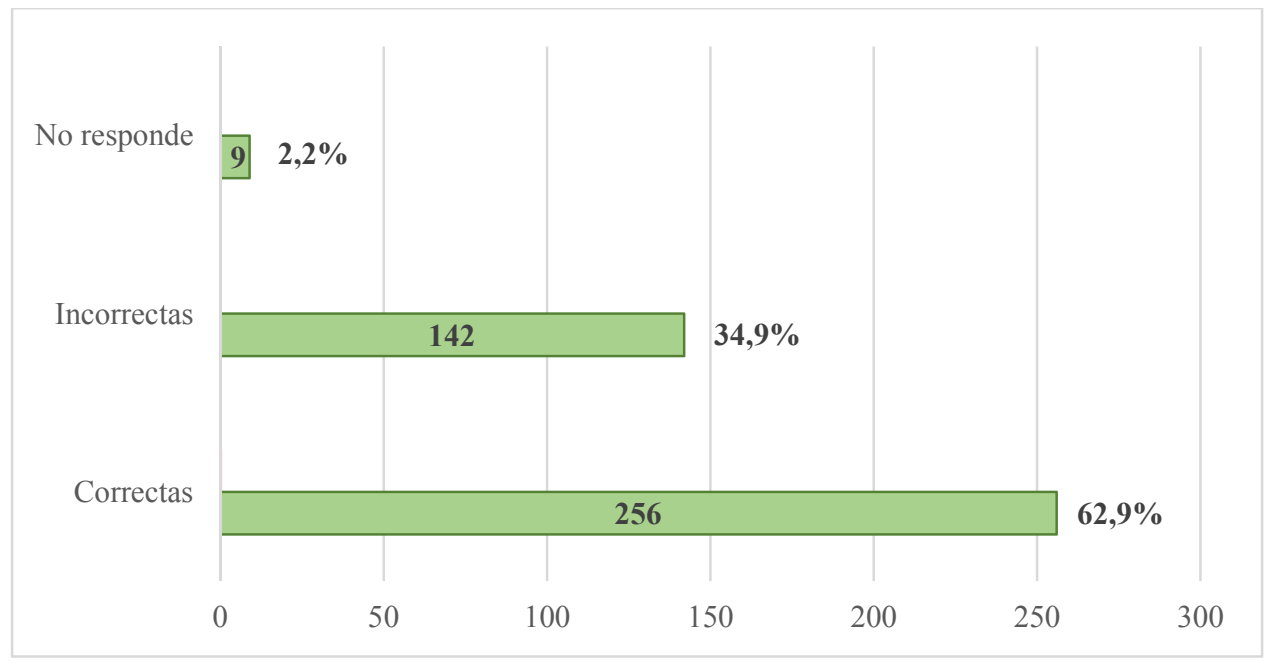

Fuente: elaboración propia

En esta pregunta, se pide a los participantes que utilicen el gráfico para producir argumentos que puedan utilizar los estudiantes del Grupo A con el propósito de convencer al profesor de que el Grupo B no tuvo necesariamente un mejor rendimiento en el examen considerado. Deben obtener una información que no está representada en el gráfico y que no se deduce con operaciones o comparaciones. De acuerdo con los parámetros PISA (INECSE, 2005), los argumentos considerados válidos pueden estar relacionados con: el número de 
estudiantes que aprueban, la influencia del caso extraño o el número de estudiantes con puntuaciones de nivel más alto, que no están reflejados en la gráfica, es decir con puntuaciones notables o sobresalientes. El $62,9 \%$ de los participantes indicaron algunos de esos argumentos o combinaciones de ellos. La Tabla 1 muestra la distribución porcentual de los argumentos considerados válidos.

Tabla 1

Argumentos válidos presentados en la pregunta tres

Argumento

Porcentaje

\begin{tabular}{l}
\hline $\begin{array}{l}\text { Más alumnos en el Grupo A que en el Grupo B aprobaron el } \\
\text { examen (1). }\end{array}$
\end{tabular}

Si ignoras al peor alumno del Grupo A, los alumnos del Grupo

A lo han hecho mejor que los del Grupo B (2)

1,0

Más alumnos del Grupo A que del Grupo B obtuvieron la puntuación de 80 o más (3).

Usa 1 y 2

1,2

Usa 1 y 3

9,3

Usa 2 y 3

0,3

Usa 1,2 y 3

0,7

Total

62,9

Fuente: elaboración propia

El argumento más utilizado está vinculado a la cantidad de aprobados en cada grupo, ya que 150 de los 255 argumentos considerados válidos entran en esa categoría. Este argumento se puede producir a partir del análisis directo del gráfico, a partir del cual los participantes señalan el número de aprobados de cada grupo y la diferencia entre ellos. Algunos ejemplos de los argumentos que integran esta categoría son:

P18: El grupo A tiene 11 aprobados, mientras que el grupo B solo tiene 10.

P74: Al sumar los estudiantes de cada barra, a partir de la calificación 50 (aprobados) hay más el grupo A, que del B.

P308: Los aprobados del grupo A son superiores por un (1) estudiante a los del grupo B. 
El segundo argumento más utilizado también proviene del análisis del gráfico para establecer diferencias entre frecuencias, esta vez de la cantidad de estudiantes con más altas calificaciones. Ejemplos de argumentos de esta categoría son:

P79: Los alumnos del grupo A pueden destacar que tuvieron un alumno más que los del grupo $\mathrm{B}$ con 80-89 puntos.

P261: Bueno, podrían argumentar que el grupo A (2 estudiantes) obtuvo una de las calificaciones más altas que grupo B (1 estudiante), siendo esta entre 80-89 puntos.

Solo cuatro participantes vincularon el gráfico con la información de sobre la media que se daba al comienzo. Este es un argumento que requiere observar que existe un caso atípico en el Grupo A, establecer su relación con la media y cómo está es afectada por valores extremos:

P118: El estudiante que sacó entre 0-9 puntos podría estar distorsionando el promedio del grupo.

P394: El estudiante del grupo A que sacó entre 0-9 es un dato atípico en la distribución de las notas d ella estudiantes. Podría argumentarse que el grupo A salió mejor debido a que tienen una media de los puntajes más elevada y con menos desviación o variación en la distribución de las notas, sin tomar en cuenta el estudiante con datos atípicos.

A diferencia de los anteriores argumentos, este no se puede producir únicamente con un análisis detallado del gráfico. Los argumentos combinados reúnen el $11,4 \%$ del total de respuestas. Generar un argumento de esta naturaleza requiere la identificación de dos aspectos distintos e interrelacionarlos, lo cual produce un argumento más sólido. Un ejemplo de la combinación de los argumentos 2 y 3 es:

P246: El grupo A es mejor si se aplica la eliminación de los outliers en este caso el estudiante que sacó la peor nota está fuera o se sale del grupo. Por otra parte, el grupo A tiene 2 estudiantes excelentes contra uno solo del grupo B.

En el siguiente argumento se combinan los tres argumentos básicos:

P277: El promedio del grupo está sesgado por una calificación anormalmente baja. El Grupo B reprobó más alumnos (si la aprobatoria es más del 50\%). La máxima calificación fue alcanzada por 2 alumnos del grupo A y solo 1 del grupo B.

La Tabla 2 muestra las categorías usadas para agrupar los argumentos considerados no válidos en la pregunta tres. El grupo mayor de argumentos considerados no validos se incluyeron 
en la categoría idiosincrático, en la clasificación de Aoyama (2007): los participantes no pueden leer valores o tendencias en gráficas, es decir, no conectan la característica extraídas de las gráficas con el contexto, como se muestra en los siguientes ejemplos:

P96: Nosotros los del grupo A obtuvimos un comportamiento uniforme con un promedio de 3 pts.

P202: Podrían explicarle los ejercicios detalladamente a ver.

P374: Los estudiantes del grupo A, pese a ser el mismo número de estudiantes que el grupo B, obtuvo un promedio de calificaciones

Tabla 2 más estable que este último.

Argumentos no válidos presentados en la pregunta tres

\begin{tabular}{lc}
\hline \multicolumn{1}{c}{ Argumento } & Porcentaje \\
\hline Idiosincrático & 18,2 \\
El Grupo B tiene mejor rendimiento & 10,8 \\
Los grupos son similares & 5,9 \\
\hline Total & 34,9 \\
\hline
\end{tabular}

Fuente: elaboración propia

Un segundo conjunto de argumentos no validos es aquellos que presentan razones para apoyar la idea que el Grupo B tiene un mejor rendimiento. Generalmente leen sólo una parte de la gráfica, comparando en los dos grupos únicamente los valores más altos:

P248: Los estudiantes del grupo B tienen mejores calificaciones que los del grupo A y se ve en el gráfico.

P198: Hay un mayor número de estudiantes del grupo $\mathrm{B}$ con calificaciones superiores a 60 puntos.

Una última colección de argumentos no validos son aquellos que señalan que los grupos son similares. Es necesario recordar que en la preguntan se solicitan argumentos que el Grupo A para demostrar al profesor que el Grupo B no tuvo el mejor rendimiento. Aquí se incluyeron argumentos como los siguientes, que no tienen en cuenta la frecuencia de cada resultado:

P38: Al sumar los resultados son parejos el A vs B.

P71: el grupo A obtuvo calificaciones 70 a 90, similares al B.

Los resultados del grupo estudiado en la Pregunta 3 son semejantes a lo reportado por Aoyama y Stephens (2003) en cuanto a las dificultades para generar una nueva información, así como con los hallazgos de Arredondo et al. (2019), Eudave (2009), Fernández et al. (2019) y 
García-García et al. (2018), en relación a los problemas para hacer extrapolaciones sobre la base del gráfico y ofrecer un argumento a favor de una posición especifica. La mayor parte de los argumentos no válidos se incluyeron en la categoría Idiosincrático (Aoyama, 2007), similares a los encontrados por Inzunsa (2015).

En la Pregunta 4 a los participantes se les presenta un gráfico elaborado por el Grupo A como evidencia de que su rendimiento es muy superior al del Grupo B, y se les pregunta si están de acuerdo con esa afirmación y que argumenten su respuesta, utilizando el gráfico. En la Figura 5 se presentan los porcentajes de respuestas alcanzados en esta pregunta.

Figura 5. Porcentaje de respuestas a la pregunta cuatro

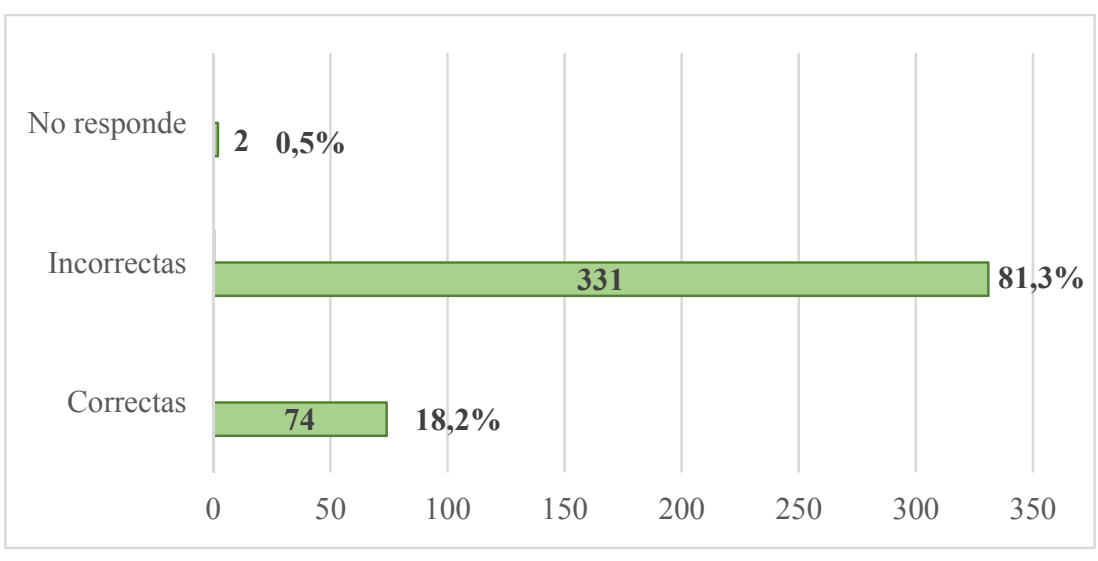

Fuente: elaboración propia

De los 151 participantes que señalan no estar de acuerdo con el Grupo A, solo 74 combinan ese desacuerdo con un argumento válido. Ello representa el 18,2\% de las respuestas proporcionadas. Los argumentos considerados válidos se agruparon en dos categorías, cuya frecuencia y distribución porcentual se muestra en la Tabla 3.

Tabla 3

Argumentos válidos presentados en la pregunta cuatro

Argumento Porcentaje

Señala diferencia porcentual entre grupos dice que no es una
gran diferencia. No le da la razón a grupo A.

No señala porcentaje, pero afirma que el rendimiento es similar o no hay gran diferencia. No le da la razón a grupo A.

Total 18,2

Fuente: elaboración propia 
Respuestas como las siguientes se incluyeron en la primera categoría. Son respuestas que tratan de especificar la diferencia porcentual entre los dos grupos para usarla como soporte de su argumento y refutar que el rendimiento del grupo A no es muy superior al del grupo B Para calcular esta diferencia, han tenido que leer la gráfica y realizar con ella comparaciones, lo cual supone el segundo nivel en la clasificación de Curcio (1989) y Friel et al. (2001):

P182: No es "muy" superior porque la diferencia entre un grupo y otro es de $0,3 \%$.

P308: El rendimiento de los estudiantes del Grupo A es superior al del Grupo B, pero por muy poco $52,09 \%$ vs $51,85 \%$;

En la segunda categoría se incluyeron los casos donde se señala que no se puede considerar muy superior sin especificar la diferencia según la escala. No queda claro en su respuesta si son conscientes del sesgo en la escala empleada:

P66: El rendimiento del grupo A es mayor pero no es "muy superior" la diferencia entre ambos grupos es muy estrecha y puede no ser significativa.

P164: No es "muy superior" es un poco mejor. Se demuestra que obtienen mejores resultados de aprobación, pero no me parece que es "muy superior".

Un total de 254 participantes de los 407 consultados $(62,4 \%)$ indicaron que estaban de acuerdo con el Grupo A y consideraban que su rendimiento era muy superior al del Grupo B. A ellos hay que agregarle 77 (18,9\%) participantes que señalaron no estar de acuerdo con el Grupo A, pero se apoyaron en argumentos considerados no válidos. Es por ello que el total de respuestas incorrectas llega a $331(81,3 \%)$. La Tabla 4 presenta, en frecuencia y porcentaje, las categorías donde se agruparon los argumentos considerados no válidos.

Tabla 4

Argumentos no válidos presentados en la pregunta cuatro

Argumento

Porcentaje

Señala diferencia porcentual entre grupos, pero da la razón al Grupo

13,5

A

Se refiere al porcentaje de forma general. Le da la razón al Grupo A $\quad$ 19,7

Idiosincrático para darle la razón al Grupo A. 20,6

Idiosincrático, pero no le da la razón al Grupo A. $\quad 18,9$ 


Señala que en el gráfico se aprecia la diferencia, da razón al Grupo A. $\quad 8,6$

Total 81,3

Fuente: elaboración propia

Un $13,5 \%$ de los participantes presenta argumentos donde señala a la diferencia porcentual entre los grupos, pero aun así apoya al Grupo A. Ejemplos de los argumentos de esta categoría son:

P69: Porque el porcentaje de los estudiantes aprobados grupo A $(52,10 \%)$ es mucho mayor que el del B $(51,85 \%)$.

P281: El 52,10\% correspondiente al grupo A es mayor al obtenido por el grupo B de 51,90.

Hay un segundo grupo que hace referencia al porcentaje de aprobados en cada grupo, sin mencionar la diferencia exacta, pero de igual forma apoyan al Grupo A, Este grupo parece leer en forma correcta el gráfico y aun así opinan que el Grupo A tiene razón. No muestran una lectura crítica de acuerdo a Aoyama (2007), puesto que no tienen en cuenta que la diferencia es muy pequeña, ni las otras posibles calificaciones, por ejemplo:

P50: El porcentaje de aprobados en el grupo A es mayor.

P309: El \% de pruebas aprobadas del grupo A es superior a \% del grupo B.

Este grupo parece leer en forma correcta el gráfico, pero los participantes opinan que el Grupo A tiene razón. La categoría que reúne mayor frecuencia es aquella donde están argumentos que apoyan al Grupo A usando razones generales, con poca o ninguna base estadística.

P30: La sumatoria de las evaluaciones del grupo A demuestra que el rendimiento ha sido mejor que el grupo B.

P32: Al promediar las Evaluaciones del grupo A da superior al único valor presentado del grupo B.

P178: Porque se verificaron por medio de cálculos totales la cantidad de estudiantes que aprobaron con mejores calificaciones para tener un resultado favorable al grupo A.

P219: La respuesta es sí ya que pudo ser que el grupo A rindió más que el grupo B en el año, una de las cosas que pueden de que el grupo $\mathrm{A}$ rindió más que el grupo $\mathrm{B}$ es porque a lo mejor los del grupo $\mathrm{B}$ o no se prepararon como el grupo A o los alumnos del grupo B no le entienden al profesor. 
Los argumentos de esta categoría más que basarse en el gráfico en sí, parecen tomar posición por empatía con el Grupo A. Ninguno menciona al gráfico o sus valores, tienen en común que se fundamentan en diversas ideas, sin mayores fundamentos, para apoyar al Grupo A. Este grupo de respuesta parece coincidir con el nivel 0, Perspectiva Personal, de la jerarquía de Fernández et al. (2019), ya que ofrece una respuesta personal sin considerar los datos del gráfico. En otra categoría se incluyeron las respuestas de los participantes que consideran el Grupo A tienen la razón, porque así se observa en la gráfica, ejemplo de este tipo de argumento son:

P108: En el gráfico se puede apreciar que es mayor el rendimiento del grupo A en el año.

P147: En el grupo A hubo más estudiantes aprobados que en el grupo B. Y en este gráfico se está tomando como referencia los estudiantes aprobados.

P380: Como podemos observar claramente en el gráfico el grupo A obtuvo mejor en toda la asignatura por lo tanto podemos asegurar que el grupo B es superior al A simplemente por tener una alta en un solo examen.

Estos argumentos parecen reflejar que los participantes se dejan llevar por el gráfico sin detallar en los valores de las escalas. El 18,9\% de los argumentos no apoyaron al Grupo A en la afirmación de que tenían un rendimiento muy superior, pero hicieron uso de argumentos que no se relacionaban con la situación o el gráfico mostrado. Algunos de esos argumentos se muestran a continuación, el último de los cuales también podría considerarse del nivel 0 de la jerarquía de Fernández et al. (2019). son:

P34: El grupo B aprobó más exámenes por encima de los 60 puntos. P107: Aprobaron más pero no significa que tengan notas más altas.

P344: No estoy de acuerdo puesto que la gráfica fue realizada por los alumnos del grupo A dónde la respectiva gráfica debe estar realizada por el profesor.

Como en el caso del trabajo de Garzón-Guerrero (2020), este grupo tuvo dificultades para superar el sesgo del gráfico y generar una interpretación adecuada. Su comportamiento parece semejante al grupo estudiado por Monteiro \& Ainley (2007), en cuanto a desdeñar la información representada en el gráfico y utilizan otros aspectos para elaborar una interpretación. Batanero (2004) señala que la cultura estadística no es únicamente conocimiento y capacidad, también involucra una parte emocional (sentimientos, valores, actitudes, entre otros) que entra 
en juego al momento de interpretar gráficos, por ejemplo. En este caso, la empatía con uno de los grupos parece haber permeado en la argumentación que hizo parte de la muestra.

\section{Diferencias de respuestas según edad y formación}

Debido a la heterogeneidad del grupo en cuanto a edades se planteó analizar las diferencias observadas en la distribución de las respuestas consideradas. El número de respuestas correctas e incorrectas clasificadas por grupo de edades se reporta en la Tabla 5. Se planteó la hipótesis de homogeneidad de las respuestas según las edades y para probarla se usó el estadístico Chi-cuadrado de Pearson, comprobando antes las condiciones de aplicación. El estadístico calculado fue 5,093 con 5 grados de libertad y con un valor $p$ de 0,532 , no siendo las diferencias observadas estadísticamente significativas para rechazar la hipótesis nula, con un nivel de significación del 5\%. Este resultado se interpreta como que, indistintamente de su edad los participantes parecen juzgar la información de los gráficos de forma similar.

Tabla 5 .

Tipo de respuesta por edades

\begin{tabular}{rccc}
\hline $\begin{array}{c}\text { Grupo } \\
\text { etario }\end{array}$ & \multicolumn{2}{c}{ Respuesta } & Total \\
& Correcta & Incorrectas & \\
\hline $\mathbf{1 7 - 2 6}$ & $\mathbf{1 8 8}$ & $\mathbf{1 5 7}$ & $\mathbf{3 4 5}$ \\
$\mathbf{2 7 - 3 6}$ & $\mathbf{1 5 3}$ & $\mathbf{1 0 3}$ & $\mathbf{2 5 6}$ \\
$\mathbf{3 7 - 4 6}$ & $\mathbf{1 9 2}$ & $\mathbf{1 2 3}$ & $\mathbf{3 1 5}$ \\
$\mathbf{4 7 - 5 6}$ & $\mathbf{1 7 8}$ & $\mathbf{1 2 8}$ & $\mathbf{3 0 6}$ \\
$\mathbf{5 7 - 6 6}$ & $\mathbf{1 7 1}$ & $\mathbf{1 3 2}$ & $\mathbf{3 0 3}$ \\
$\mathbf{6 7 - 8 6}$ & $\mathbf{4 7}$ & $\mathbf{4 5}$ & $\mathbf{9 2}$ \\
\hline Total & 929 & 688 & 1617 \\
\hline
\end{tabular}

Fuente: elaboración propia.

De manera similar se analizó la distribución de respuestas al cuestionario según el máximo nivel de formación alcanzado (Tabla 6). En este caso el valor encontrado de Chicuadrado de Pearson con 5 grados de libertad fue 5,095 con un valor p de 0,079. En consecuencia, las diferencias observadas no son estadísticamente significativas con un nivel de significación del 5\%, lo que se interpreta como que la proporción de respuestas correctas e incorrectas no difieren por el nivel de formación alcanzado por los participantes. Este resultado 
no parece corresponderse con lo reportado en la literatura, según la cual, los estudiantes que se inician en el estudio de los gráficos estadísticos tienden a centrarse en las características locales del gráfico, mientras que los expertos tienden a ver un gráfico de forma global (Khalil, 2005), por lo cual lo analizan de forma apropiada.

Tabla 6.

Tipo de respuesta por niveles de formación alcanzado

\begin{tabular}{|c|c|c|c|}
\hline \multirow{2}{*}{ Sección } & \multicolumn{2}{|c|}{ Respuesta } & \multirow{2}{*}{ Total } \\
\hline & Correcta & Incorrectas & \\
\hline Doctorado & 111 & 65 & 176 \\
\hline Maestría & 155 & 119 & 274 \\
\hline Especialización & 246 & 162 & 408 \\
\hline $\begin{array}{l}\text { Universitaria sin } \\
\text { postgrado }\end{array}$ & 220 & 160 & 380 \\
\hline Universitaria incompleta & 197 & 182 & 379 \\
\hline Total & 929 & 688 & 1617 \\
\hline
\end{tabular}

Fuente: elaboración propia.

Un punto a destacar es el alto número de respuestas en todas las preguntas, el cual osciló entre $100 \%$ y el 98,5\%. Esto podría indicar que los participantes apreciaron la situación, gráfico y contexto, como familiar, al punto de poder ofrecer las respuestas correctas. En situaciones cotidianas, esa confianza podría llévalos a tomar decisiones sobre la base de gráficos manipulados o mal elaborados, llevándolos a conclusiones erróneas, como en el caso de la pregunta cuatro.

\section{Consideraciones finales}

Aunque no son generalizables, los resultados expuestos aportan información sobre cómo un grupo de ciudadanos venezolanos comprenden e interpretan gráficos estadísticos, específicamente el gráfico de barras; cuestión que no había investigado en Venezuela, de acuerdo con la revisión bibliográfica realizada. El grupo estudiado, de distintos niveles de formación universitaria y diferentes edades, mostró dificultades en las actividades de mayor exigencia en el análisis de gráficos. Eso concuerda con lo observado en estudios precedentes 
realizados en otros países. Todo ello ratifica que producir información o hacer análisis crítico de un gráfico requiere de mucho trabajo desde la escuela.

Al momento de estudiar gráficos estadísticos, los planes y programas de estudios venezolanos hacen énfasis en su construcción, dando poco espacio a la interpretación o al estudio de gráficos con sesgos. Este enfoque podría estar poniendo en desventaja al ciudadano venezolano al momento de enfrentar gráficos en los medios de comunicación o redes sociales. Dos de las funciones de los gráficos son: comunicar información e instrumento para analizar datos. Si los ciudadanos no pueden cumplir a cabalidad alguna de las dos, entonces parece necesario un cambio de orientación en el estudio de los gráficos. Se debe trabajar más para qué sirven, cuáles convenciones necesitan cumplir y cómo extraer información para alcanzar altos niveles en su comprensión. Así mimo parece recomendable que la escuela le preste más atención al caso de los gráficos erróneos y estudiar con más detalle la construcción e interpretación de las escalas (Cavalcanti \& Guimarães, 2019).

Lo más probable es que sea necesario prestar más atención a la formación de los docentes en aspectos relacionados con la estadística y, en particular, con los gráficos; ya que las investigaciones, aquí señaladas, sugieren que los docentes (activos y en formación) suelen tener dificultades y errores en la interpretación de gráficos. Sugerimos que los docentes trabajen con gráficos en el marco del análisis de datos, comprendan cómo se usan en diferentes áreas y como se puede extraer información, y analicen gráficos publicados en medios de comunicación.

Todo ello debe coadyuvar mejorar la compresión de los gráficos y agregar conocimientos y habilidades que le permitan desenvolverse forma eficiente en la sociedad actual, la cual requiere de ciudadanos activos que apuntalen los procesos democráticos.

\section{Agradecimiento}

Los autores agradecemos a todas las personas que cedieron parte de su tiempo para dar respuesta a nuestro cuestionario. De igual forma agradecemos a los árbitros y a la Dra. Carmen Batanero por sus observaciones y comentarios, los cuales ayudaron a mejorar la versión inicial de nuestro trabajo. El autor Audy Salcedo agradece el apoyo que le brinda del Institute of International Education's Scholar Rescue Fund (IIE-SRF). 


\section{Referencias}

Aoyama, K. (2007). Investigating a hierarchy of students' interpretations of graphs. International Electronic Journal of Mathematics Education, 2, (3), 298-318.

Aoyama, K. \& Stephens, M. (2003). Graph interpretation aspects of statistical literacy: A Japanese perspective. Mathematics Education Research Journal, 15(3), 207-225.

Arredondo, E. H., García-García, J. I., \& López, C. (2019). Niveles de lectura de estudiantes de licenciatura: el caso de una tabla y una gráfica de líneas. Revista digital Matemática, Educación e Internet, 19(2), 1-13. https://doi.org/10.18845/rdmei.v19i2.4214

Batanero, C. (2004). Los retos de la cultura estadística. Yupana, 1(1), 27-37. https://doi.org/10.14409/yu.v1i1.238.

Batanero, C. \& Borovcnik, M. (2016). Statistics and probability in high school, Rotterdamn. The Netherlands: Sense Publishers.

Batanero, C., Arteaga, P., \& Ruiz, B. (2010). Análisis de la complejidad semiótica de los gráficos producidos por futuros profesores de educación primaria en una tarea de comparación de dos variables estadísticas. Enseñanza de las Ciencias, 28(1), 141-154. Disponible en: https://www.raco.cat/index.php/Ensenanza/article/view/189102

Ben-Zvi, D. \& Makar, K. (2016). International perspectives on the teaching and learning of statistics. En D. Ben-Zvi y K. Makar (Eds.). The Teaching and Learning of Statistics. International Perspectives (pp. 1-10). New York: Springer.

Cavalcanti, M. \& Guimarães, G. (2019). Compreensão de escala representada em gráficos por crianças e adultos em início de escolarização. Jornal Internacional de Estudos em Educação Matemática, 12(2), 207-220.

Common Core State Standards Initiative (CCSSI) (2010). Common Core State Standards for Mathematics. Washington, DC: National Governors Association for Best Practices and the Council of Chief State School Officers.

Curcio, F. R. (1987). Comprehension of mathematical relationships expressed in graphs. Journal for Research in Mathematics Education, 18(5), 382-393.

Curcio, F. R. (1989). Developing graph comprehension. Reston, VA: NCTM.

Eudave, D. (2009). Niveles de comprensión de información y gráficas estadísticas en estudiantes de centros de educación básica para jóvenes y adultos de México. Educación Matemática, 21(2), 5-37.

Fernández, N., García-García, J. I., Arredondo, E., \& López, C. (2019). Comprensión de una tabla y un gráfico de barras por estudiantes universitarios. Areté, 5 (10), 145-162.

Franklin, C., Kader, G., Newborn, D.S., Moreno, J., Peck, R., Perry, M., \& Schaeffer, R. (2005), A Curriculum Framework for pre K-12 Statistics Education. Disponible en: http://www.amstat.org/education/gaise/.

Friel, S. N., Curcio, F. R., \& Bright, G. W. (2001). Making sense of graphs: critical factors influencing comprehension and instructional implications. Journal for Research in Mathematics Education, 32(2), 124-158. https://doi.org/10.2307/749671 
Gal, I. (2002). Adults' statistical literacy: meanings, components, responsibilities. International Statistical Review, 70(1), 1-51. https://doi.org/10.1111/j.1751-5823.2002.tb00336.x

García-García, J. I., Calvario, C. L. \& Arredondo, E. H. (2018). Interpretación de una tabla y una gráfica circular por estudiantes de licenciatura. TANGRAM, 1(3), 24-39. https://doi.org/10.30612/tangram.v1i3.8298

Garfield, J. \& Ben-Zvi, D. (2007). How students learn statistics revisited: A current review of research on teaching and learning statistics. International Statistical Review, 75(3), 372396. https://doi.org/10.1111/j.1751-5823.2007.00029.x.

Garzón-Guerrero, J.A. (2020). Lectura e interpretación de gráficos estadísticos de la prensa escrita en futuros profesores: un estudio exploratorio en Italia y Portugal. En M. M. Gea. R. Álvarez-Arroyo y J.A. Garzón (Eds.), Seminario Hispano Brasileño de Educación Estadística. Granada: Grupo PAI FQM-126.

Gea, M., Arteaga, P., \& Cañadas, G. (2017). Interpretación de gráficos estadísticos por futuros profesores de Educación Secundaria. Avances de Investigación en Educación Matemática, 12, 19-37. https://doi.org/10.35763/aiem.v1i12.189

Guardia de Sanz, A. (1988). Historia de la Facultad de Ciencias Económicas y Sociales de la Universidad Central de Venezuela 1938-1958. Caracas: Ediciones FaCES, UCV.

Hernández, R., Fernández, C., \& Baptista, P. (2006). Metodología de la investigación. México: McGraw Hill.

Instituto Nacional de Evaluación y Calidad del Sistema Educativo (INECSE) (2005). PISA 2003. Pruebas de matemáticas y de solución de problemas. Madrid: Ministerio de Educación y Ciencia.

Inzunsa, S. (2015). Niveles de interpretación que muestran estudiantes sobre gráficas para comunicar información de contextos económicos y sociodemográficos. Revista Mexicana de Investigación Educativa, 20(65), 529-555.

Khalil, K.A.I. (2005). Expert-novice differences: Visual and verbal responses in a two-group comparison task Tesis de Máster. University of Massachusetts, Amherst, MA, USA. https://scholarworks.umass.edu/theses/2428

Leavy, A. (2006). Using data comparison to support a focus on distribution: Examining preservice teacher's understandings of distribution when engaged in statistical inquiry. Statistics Education Research Journal, 5(2), 89-114.

Ministerio de Educación. (1987). Programa de estudio y manual del docente. Tercera etapa. Educación básica. Asignatura matemática - física. Caracas: Autor.

Ministerio de Educación (1990). Programa de articulación del nivel de educación media diversificada y profesional. Asignatura matemática. Primero y segundo año. Caracas: Autor.

Ministerio de Educación (1997). Currículo Básico Nacional. Programa de Estudio de Educación Básica. Caracas: Autor.

Monteiro, C. \& Ainley, J. (2006). Student teachers interpreting media graphs. En A. Rossman, \& B. Chance (Eds.), Proceedings of the Seventh International Conference on Teaching Statistics. Salvador, Brazil: International Statistical Institute and International Association 
for Statistical Education. Disponible en: http://iaseweb.org/documents/papers/icots7/2G1_MONT.pdf?1402524964

Monteiro, C. \& Ainley, J. (2007). Investigating the interpretation of media graphs among student teachers. International Electronic Journal of Mathematics Education 2(3), 187-207.

Salcedo, A. (2017). Gráficos estadísticos en libros de texto para educación primaria de Guatemala y Venezuela. Educação Matemática Pesquisa, 18(3), 1141-1163.

Sutherland, S. \& Ridgway, J. (2017). Interactive visualisations and statistical literacy. Statistics Education Research Journal, 16(1), 26-30.

Tukey J. W. (1977). Exploratory data analysis. Reading, MA: Addison-Weseley.

Weiland, T. (2017). Problematizing statistical literacy: An intersection of critical and statistical literacies. Educational Studies in Mathematics, 96(1), 33-47.

Wu, Y. (2004). Singapore secondary school students' understanding of statistical graphs. Trabajo presentado en 10th International Congress on Mathematics Education (ICME-10). Disponible en http://iase-web.org/documents/papers/icme10/Yingkang.pdf.

\section{Autores}

Audy Salcedo

Licenciado en Educació,n Mención Matemáticas (UCV). Magister en Enseñanza de la Matemática (UPEL IPC). Doctor en Educación (UCV). Profesor Titular Departamento de Estadística e Informática en la Escuela de Educación, Universidad Central de Venezuela. Línea de Investigación: Educación matemática y educación estadística.

E-mail: audy.salcedo@ucv.ve

Jesús A. González U. Licenciado en Ciencias Estadísticas (UCV), Especialista en Análisis de Datos en Ciencias Sociales (UCV). DEA en Planificación de la Educación (IIPE-UNESCO). DocenteInvestigador y Jefe de Departamento de Estadística e Informática Aplicada a la Educación (Escuela de Educación- UCV). Líneas de Investigación: Evaluación institucional, y rendimiento estudiantil. E-mail: jagonza05@gmail.com

Johnnalid González Licenciado en Ciencias Estadísticas (UCV). Profesora de estadística aplicada a la educación (UCV y Universidad Monte Ávila). Diplomado en Componente Docente (UCV), Marketing Digital (Instituto Internet) y Diseño y Tutoría Virtual (UCV). Línea de Investigación: Gerencia educativa en entornos virtuales. E-mail: estadisticaucv.2012@gmail.com 